\title{
Characterization and Genetic Variability of Indian Mustard Genotypes for Branching Behavior, Yield and Its Attributes under Rainfed Condition
}

\author{
Khushboo Chandra*, Anil Pandey and S.B. Mishra \\ Department of Plant Breeding and Genetics, Dr. Rajendra Prasad Central Agricultural \\ University, Pusa (Samatipur) Bihar - 848125, India \\ *Corresponding author
}

\section{Keywords}

Brassica juncea L., Basal branching, Variability, Heritability, Metroglyph, Ideotype

Article Info

Accepted:

04 May 2018

Available Online:

10 June 2018

\section{A B S T R A C T}

An experiment was conducted in Randomized Complete Block Design (RCBD) for characterization based on branching behavior, genetic variability and heritability studies on Indian mustard (Brassica juncea L. Czern \& Coss), accommodating 50 genotypes, from various Rapeseed and Mustard centres located across country, randomly in three replications during Rabi 2015-16 at the research farm of Tirhut College of Agriculture, Dholi, Muzaffarpur under rainfed condition. Branching behaviour and grain yield categorized 50 genotypes in 9 categories viz; BB-HY (1), BB-MY (11), BB-LY (1), MNbb-HY (1), M-Nbb-MY (25), M-Nbb-LY (1), H-Nbb-MY (6), H-Nbb-LY (6) and none of the genotype in H-Nbb-HY category. Five high yielding genotype reflecting different branching patterns were Rajendra Suphlam (BB), Pusa Mahak (Nbb), NDRE7 (BB), NRCDR-2 and RH0819 (both Nbb). Based on morphological characterization under rainfed condition Rajendra Suphlam reflected superiority for 14 out of 25 metric traits for flowering, maturity, plant height, root, shoot, siliqua and TSW followed by Pusa Mahak for 6 traits involving flowering, primary and secondary branches, NS, HI and DME. Kanti for 6 traits including short internode, HP, HFPB, HFS AB and AS, whereas KMR-10-1 for oil content. High heritability coupled with high GAM for NS, HP, HFPB, HFS and HI indicated predominance of additive gene action and were least influenced by the environment. Suggesting Brassica juncea ideotype contributing to the highest yield in a specified environment ie under resource constrained rainfed situations (only basal dose of RDF and residual moisture condition), the study indicated importance of lower positioned primary basal branches accommodating at least two secondary branches per PBP, less number of primary and secondary Nbb, lower HFS, medium-tall plant, superior in root volume, more no. of siliqua, HI and DME.

\section{Introduction}

Mustard has been grown in the Indian subcontinent as an oilseed crop. Brassica species are one of the major oilseed crops of the world (Md. Mukhlesur et al., 2004). It is the second largest oilseed crop in India after groundnut.
Indian mustard (Brassica juncea L.), locally called as Laha, Raya or Rai, belongs to family Cruciferae; genus Brassica (Iraddi, 2008) and introduced as an oily herb, which is appropriate for zones with short seasons and less rainfall (Burton et al., 1999). It is cultivated in winter season and is a thermo as 
well as photosensitive crop (Ghosh and Chatterjee, 1998). Indian mustard [B. juncea (L.) Czern Coss.] is the most important member of the oilseeds and accounts for about 70 per cent of the area under rapeseed \& mustard which is still cultivated in large acreage as rainfed crop under resource - poor condition on farmers' fields. Water stress is another important abiotic factor (Campbell et al., 1992).The effect of drought stress is a function of genotype, intensity and duration of stress, weather conditions, growth, and developmental stages of rapeseed (Robertson and Holland, 2004).

The success of plant breeding programs relies heavily on the existence of genetic variability in plants for particular traits. Cultivar depends upon the nature and magnitude of variation for different yield components, the assessment of genetic parameters like phenotypic coefficient of variation (PCV), genotypic coefficient of variation $(\mathrm{GCV})$, heritability $\left(\mathrm{h}^{2}\right)$ and genetic advance (GA\%) is a pre-requisite for making effective selection. The estimation of genotypic co-efficient of variation indicates the amount of genetic variation presents for different traits while heritability gives as insight into the proportion of the variation which is inherent.

For successful utilization of genetic variability crop breeders emphatically search for the traits of importance and subsequently to incorporate it genetically into a usable variety. Moreover plant ideotype of Brassica, possessing desirable component traits, contributing towards yield in a specified (here rainfed condition) environment could provide a basis for sustainable Brassica improvement (Donald, 1962a\&b). It can also provide a valuable guide to breeders particularly hybridization followed by pedigree selection for varietal development under rain dependent/residual moisture critical environment. Genetic diversity is the pre- requisite for hybridization programme to obtain desirable genotypes and it comprises new land races, local selection, elite cultivars and exotic germplasm of crop plants. Genetic diversity is very much essential to meet the diverse goals in plant breeding such as for producing cultivars with increased yield (Joshi and Dhawan, 1966), wider adoption, desirable quality and pest resistance (Nevo et al., 1982).

\section{Materials and Methods}

The experiment was laid out in Randomized Complete Block Design (RCBD) with three replications during Rabi season (2015-16) and was planted on $10^{\text {th }}$ October 2015 under rainfed condition consisting of 50 Indian mustard genotypes, including four checks namely, Pusa Mahak (Zonal Check), Varuna (National Check), Pusa Bold (National Check) and Rajendra Suphlam (Local Check) for branching behaviour, variability and heritability study, received from differentAll India Co-ordinated Research ProjectRapeseed and Mustard centres: DRMR, Bharatpur, Rajasthan, CCSHAU, Hisar, Haryana, BARC, Trombay, Maharastra, GBPUAT, Pantnagar, Uttarkhand, CSAUAT, Kanpur, U.P, IARI, New Delhi, ARS, RAU, Sriganganagar, Rajasthan, DR.RPCAU, Dholi, Bihar, NDUAT, Faizabad, U.P and BAU, Kanke, Ranchi, Jharkhand, providing only basal dose of fertilizers i.e. $\mathrm{N}: \mathrm{P}_{2} \mathrm{O}_{5}: \mathrm{K}_{2} \mathrm{O}: \mathrm{S}:$ : 40:40:40:40 $\mathrm{kg} / \mathrm{ha}$ under residual moisture conditions after the harvest of preceeding medium early (110-115 days) paddy variety, Rajendra Bhagwati at the research farm in Loam soil $(8.4 \mathrm{pH})$ of Tirhut College of Agriculture, Dholi, Muzaffarpur $\left(25.5^{\circ} \mathrm{N}\right.$, $85.4^{0} \mathrm{E}$ and $52.12 \mathrm{~m} \mathrm{MSL}$ ) (Dr. Rajendra Prasad Central Agricultural University, Pusa), Bihar. Each plot was consisted four rows of $5.0 \mathrm{~m}$ length keeping row to row and plant to plant distance $30 \mathrm{~cm}$ and $10 \mathrm{~cm}$, respectively. The spacing between plants was maintained at $10 \mathrm{~cm}$ by thinning at 14 DAS. 
Meteorological data (Kharif \& Rabi 2015-16) reflected that the experiment was sown, under residual moisture condition, as the preceeding Kharif crop rice has received $697.20 \mathrm{~mm}$ rainfall distributed in 25 rainy days between June to September $\left(23^{\text {rd }}\right.$ to $38^{\text {th }}$ meteorological weeks 2015). After that experiment at all its phenological stages of Indian mustard crop has not received any rainfall.

The observations were recorded for days to first flower open (DFFO), days to $50 \%$ flowering (DFF), days to physiological maturity (DPM), primary branches plant $^{-1}$ $\left(\mathrm{PBP}^{-1}\right)$, secondary branches plant ${ }^{-1}\left(\mathrm{SBP}^{-1}\right)$, number of siliqua plant $^{-1}$ (NS), length of siliqua (LS), stem girth (SG), internode length (IL), height of the plant (HP), number of siliqua on primary mother axis (SPMA), height of first primary branch (HFPB), height of first siliqua (HFS), angle of branch (AB), angle of siliqua (AS), number of seeds siliqua $^{-1}\left(\mathrm{SS}^{-1}\right)$, root volume (RV), root length (RL), root girth (RG), 1000 seed weight (TSW), biological yield (BY), harvest index (HI), oil content (OC), dry matter efficiency (DME) and grain yield /plant $\left(\mathrm{GYP}^{-1}\right)$.

The data were recorded on five randomly selected plants from each genotype in each replication leaving the border rows to avoid the sampling error. The observations were recorded using standard methodology. Readings from five plants were averaged replication-wise and the mean data was subjected to statistical analyses for yield and its morpho-physio-quality traits.

The phenotypic variance was partitioned into genotypic and environmental variances for a clear understanding of the pattern of variations. The GCV, PCV, heritability, genetic advance, GA as percent of mean were calculated following standard statistical methods (Burton, 1952; Lush, 1949; Burton and Devane, 1953 and Johnson et al., 1955).

\section{Results and Discussion}

From the study of 50 Indian mustard genotypes for characterization based on branching behavior and genetic variability (Fig. 1), were categorized as basal branching genotypes (13 Genotypes having HFPB up to $30 \mathrm{~cm}$ from the base of the plant) and 37 as non-basal branching genotypes having $>30 \mathrm{~cm}$ HFPB. Among them, 27 genotypes grouped as medium non- basal branching (M-Nbb) and 10 genotypes as high non- basal (H-Nbb) branching (first primary branch placed more than $30 \mathrm{~cm}$ and $60 \mathrm{~cm}$ for respective categories from the base of the plant). It was evident that glyph placement (Fig. 2a and b) for height of first primary branch (x-axis) and yield per plant (y-axis) reflected 1 basal branching genotype with high yield (BB- HY), 11 basal branching genotypes with Medium Yield (BBMY), 1 basal branching genotype with low yield (BB-LY), 1 medium non-basal branching genotypes with high yield (M-NbbHY), 25 medium non-basal branching genotypes with medium yield (M-Nbb- MY), medium non- basal branching genotypes with low yield (M-Nbb - LY), no genotypes in high non-basal branching genotypes with medium yield (H-Nbb- MY), 6 High Nonbasal branching genotypes with Medium Yield (H-Nbb- MY), 4 High Non-basal branching genotypes with Low Yield (H-Nbb - LY).

Analysis of variance (Table 1) revealed the existence of significant differences for all the 25 traits among studied genotypes suggesting presence of sufficient variations among them in the basal and non- basal branching genotypes. The interaction between basal branching and non-basal branching genotypes were highly significant for traits namely $\mathrm{SBP}^{-}$ 1, NS, SG, IL, HP, SPMA, HFPB, HFS, AB, AS, RV and RL reflecting the scope to exploit variability exhibited by basal vs non-basal Brassica genotypes. Mean, range and coefficient of variation for 25 characters 
(Table 2 and 3) in 50 basal and non - basal branching mustard genotypes exhibited considerably wide range of variation among genotypes for studied traits except oil content. Whereas, for oil content it was narrow variability indicating sufficient diversity that is inherent among the Brassica juncea genotypes. Morphological characterization under rainfed condition revealed overall superiority of Rajendra Suphlam for 14 out of 25 metric traits, namely earliest DFFO, early DFF, early DPM, LS, SG, HP, SPMA, SS ${ }^{-1}$, RV, RL, RG, TSW, BY and GYP ${ }^{-1}$; followed by PusaMahak for 6 traits i.e. DFF, $\mathrm{PBP}^{-1}$, $\mathrm{SBP}^{-1}$, NS, HI and DME; Kanti also for 6 traits less IL, short HP, HFPB, HFS, AB\&AS; and KMR-10-1 for one attribute (high OC). Among these genotypes, only Rajendra Suphlam is basal branching whereas, PusaMahak and Kanti were M-Nbb and KMR10-1 was $\mathrm{H}-\mathrm{Nbb}$ genotype reflecting importance of all these branching patterns in terms of superiority for one or more distinguishing and/or yield attributing characters. As flowering and maturity behaviour is very important under rainfed system the otherwise desirable genotypes like Kanti (late DFF, late DPM, low DME and low $\mathrm{GYP}^{-1}$ ) and RH0116 (late DFFO, low BY) can be improved through suitable parents by hybridization - selection breeding programme. Likewise Kanti, a suitable genotype for 6 above mentioned traits reflected inferiority, under rainfed condition, for 12 attributes (lowest NS, LS, SG, HP, SPMA, SS ${ }^{-1}$, RV, RL, RG, TSW, HI, DME) and ultimately $\mathrm{GYP}^{-1}$ can also be a parent for superior traits under rainfed condition.

Thirteen basal branching genotypes were characterized for $\mathrm{PBP}^{-1}, \mathrm{SBP}^{-1}$, NS, IL and TSW, as they have both: basal $(<30 \mathrm{~cm})$ and non-basal $(>30 \mathrm{~cm})$ types of branches. From the perusal of Table 4 it was evident that $\mathrm{PBP}^{-1}$ were more on non- basal branches (Nbb) than on basal branches (BB) quantitatively varying from genotype to genotype level. $\mathrm{SBP}^{-1}$ was more on $\mathrm{BB}$ as compared to $\mathrm{Nbb}$ except in $\mathrm{TM}-2$ where $\mathrm{SBP}^{-1}$ on $\mathrm{Nbb}$ was more than in $\mathrm{BB}$. Highest number of $\mathrm{PBP}^{-1}$ and $\mathrm{SBP}^{-1}$ were found in $\mathrm{BB}$ genotype Rajendra Suphlam followed by NDRE7, BAUM-08-56 and KMR-10-2. On $\mathrm{Nbb}$, very high number of total siliqua observed in comparison to that on $\mathrm{BB}$ and its quantum varying from genotype to genotype. Slight variation in IL was observed with more length on Nbb. TSW reflected bolder seeds on $\mathrm{BB}$ in comparison to that on $\mathrm{Nbb}$ differing from genotypes to genotypes. Overall based on significant superiority of studied $13 \mathrm{BB}$ genotypes against national check Varuna it can be concluded that Rajendra Suphlam exhibited significant superiority for $\mathrm{PBP}^{-1}$, $\mathrm{SBP}^{-1}$, NS, IL and TSW for both BB and Nbb followed by NDRE7 and BAUM-08-56 for $\mathrm{SBP}^{-1}$ and NS for both $\mathrm{BB}$ and $\mathrm{Nbb}$ but for low IL NDRE7 (both BB and Nbb) and BAUM08-56 only on BB.KMR-10-2 showed superiority for $\mathrm{SBP}^{-1}$, NS, low IL and TSW on BB.

High yield is the foremost important objective for crop breeders working with any crop species like Indian mustard. Due to its polygenic inheritance it depends upon various morpho-physio-quality traits as shown in metroglyph graph (Fig. 2a and b, Tables 3 and 5). Glyph placement exhibited 9 categories accommodated as 1. BB-HY (one genotype), 2. BB-MY (11), 3. BB-LY (1), 4. M-Nbb-HY (1), 5. M-Nbb-MY (25), 6. M-Nbb-LY (1), 7. H-Nbb-HY (No genotype), 8.H-Nbb-MY (6) and 9.H-Nbb-LY (4) accommodating all the 50 genotypes. These glyphs have further accommodated 6 characteristics namely DFF, DPM, RL, RV, HFS and SPMA as arrays with high, medium and low values reflected as large, medium and short arrays separately at different angles assigned to the characters (Table 5) revealed that high yielding genotypes are Rajendra Suphlam and Pusa 
Mahak (more than 28.33g per plant) closely followed by NDRE7, NRCDR-2 and RH0819. The studied material (metroglyph) reflected variability for all the three categories i.e. early medium and late for DFF and DPM; deep, medium and short for RL; high, medium and low for RV and SPMA whereas for HFS very less variability noticed among studied genotypes as only one genotype i.e. Rajendra Suphlam $(35.67 \mathrm{~cm})$ reflected least height $(35.67 \mathrm{~cm})$ of first siliqua from base of the plant where as all other entries reflected moderate height. However, NDRE7, RH0819 and RH 8701 were very close $(36 \mathrm{~cm})$ to Rajendra Suphlam for HFS. Rajendra Suphlam reflected earliness in DFF \&DPM; deep tap root system, high RV, least HFS (placed from the base of the plant) along with high SPMA. Another BB-MY genotype, NDRE7 was similar in all these traits except moderate HFS. National check Pusa Mahak which is a Nbb-HY genotype was early in DFF \& DPM with deep tap root, high RV and high number of SPMA. Other early DFF with deep root length, high RV and high SPMA Nbb-MY genotypes were NRCDR-2, RH 8701, RH0819, TM151, Maya, DRMR 15035. TPM 128 and KMR-10-1 (both Nbb-MY genotypes) were early in DFF, with deep tap root system and high RV.

These genotypes also exhibited high per se performance for one or more (Rajendra Suphlam for 14, NDRE7 for 12, Pusa Mahak for 11, NRCDR-2 for 9, DRMR-150-35 for 6, TM151 and RH 8701 for 5, Maya and TPM 128 for 4, Divya for 3; RH0116, PM-25, RH0701, Kanti, RAURD-214, RH0819 and KMR-10-2 for 2 traits. Whereas, only for one trait DRMR LEJ 902, TM-4, RGN-13, TM-2, RAURD (E) 1002, RGN-48, RH8814 and KMR 10-1 recorded high per se. Promising medium yield genotypes like NDRE7 and PusaMahak exhibited common significantly high per se performance for $\mathrm{PBP}^{-1}, \mathrm{SBP}^{-1}, \mathrm{NS}$, LS, SG, IL, HP, RG, TSW, HI, DME additionally superior per se performance $\mathrm{SS}^{-1}$ reflected by NDRE7 whereas, NRCDR-2 (all above mentioned except TSW ; and DRMR150-35 except (except $\mathrm{PBP}^{-1}$, IL, HP, RG and TSW). Overall, basis it can be concluded that total number of branches $\left(\mathrm{PBP}^{-1}+\mathrm{SBP}^{-1}\right)$, NS, LS, RG, TSW, HI and DME played important role in medium-yielding basal/non basal branching genotypes which lacks in superiority for branch and siliqua angle which might played additional role to express high yield superiority in Rajendra Suphlam. HFS (from the base of the plant) might have played important role in making efficient plant type under rainfed condition (in addition to basal branching behaviour with more primary and secondary branches on basal branching as in RajendraSuphlam) also in medium yield genotype basal/ non- basal branching genotypes like NDRE7 (BB); RH 0819 and RH 8701 (Nbb) which reflected closely followed Rajendra Suphlam as evident from the perusal of metroglyph diagram (Fig.2a and b). Except Rajendra Suphlam, 7 genotypes namely RAURD 212, PM 25, Divya, Rohini, RH0701, Kanti and RAURD 214 were suitable with high per se performance for unique characteristic feature studied i.e. AB and AS. It is noteworthy that as siliqua characteristics namely number of siliqua, length of siliqua and seeds per siliqua developed at phenological crop stages in sequence one or other has positive or negative effect among them and also on TSW and /or on yield. So, cautious plant breeding approach is essential. None of the genotype reflected superiority for oil content which might be due to criticality of the environment at the time of oil synthesis stage and near physiological maturity terminal stage meteorological parameters. In support high influence of nonheritable non- fixable component of variation i.e. environment is highly influencing this character ultimately reflecting in low heritability and GA under selection under rainfed situation of crop Brassicas in particular Indian mustard. 
Table.1 Analysis of variance for 25 characters in Indian mustard genotypes under rainfed condition

\begin{tabular}{|c|c|c|c|c|c|c|c|}
\hline \multirow[t]{2}{*}{ No. } & \multirow[t]{2}{*}{ Characters } & \multicolumn{6}{|c|}{ Mean sum of squares } \\
\hline & & $\begin{array}{l}\text { Replication } \\
\quad(\text { d.f.=2) }\end{array}$ & $\begin{array}{l}\text { Treatments } \\
(\text { d.f. }=49)\end{array}$ & $\begin{array}{l}\text { Basal Branching } \\
\text { treatments }(B B)(\text { d.f.=12) }\end{array}$ & $\begin{array}{c}\text { Non - Basal Branching } \\
\text { treatments }(\text { NBB })(\text { d.f. }=36)\end{array}$ & $\begin{array}{c}\text { Interaction BB VS } \\
\text { NBB }(\text { d.f.=1) }\end{array}$ & Error \\
\hline 1 & DFFO & 4.29 & $11.42^{* *}$ & $11.18^{* *}$ & $11.82^{* *}$ & 0.05 & 2.78 \\
\hline 2 & DFF & 0.78 & $15.15^{* *}$ & $16.69^{* *}$ & $15.05^{* *}$ & 0.38 & 1.21 \\
\hline 3 & DPM & 11.58 & $16.05^{* *}$ & $19.26^{* *}$ & $15.39^{* *}$ & 1.47 & 4.55 \\
\hline 4 & $\mathbf{P B P}^{-1}$ & 0.52 & $8.52^{* *}$ & $11.71^{* *}$ & $7.67^{* *}$ & 0.60 & 0.29 \\
\hline 5 & SBP $^{-1}$ & 0.75 & $83.85^{* *}$ & $104.38^{* *}$ & $77.57^{* *}$ & $63.74^{* *}$ & 1.29 \\
\hline 6 & NS & 553.85 & $107908.31^{* *}$ & $123656.67^{* *}$ & $104485.09^{* *}$ & $42163.56^{* *}$ & 1610.85 \\
\hline 7 & $\mathbf{L S}$ & 0.07 & $0.56^{* *}$ & $0.91^{* *}$ & $0.45^{* *}$ & 0.15 & 0.06 \\
\hline 8 & SG & 0.09 & $6.92^{* *}$ & $8.55^{* *}$ & $6.55^{* *}$ & $0.39^{*}$ & 0.09 \\
\hline 9 & IL & 0.45 & $11.10^{* *}$ & $17.26^{* *}$ & $9.23^{* *}$ & $4.67^{* *}$ & 0.62 \\
\hline 10 & HP & 442.92 & $1729.70^{* *}$ & $2273.93^{* *}$ & $1574.46^{* *}$ & $787.81^{*}$ & 145.64 \\
\hline 11 & SPMA & 8.18 & $121.66^{* *}$ & $216.42^{* *}$ & $91.94^{* *}$ & $54.54^{* *}$ & 5.16 \\
\hline 12 & HFPB & 10.54 & $1038.11^{* *}$ & $63.59^{* *}$ & $389.11^{* *}$ & $36029.29^{* *}$ & 19.65 \\
\hline 13 & HFS & 22.08 & $1298.88^{* *}$ & $1484.99^{* *}$ & $1264.13^{* *}$ & $316.65^{*}$ & 74.24 \\
\hline 14 & AB & 19.33 & $129.98^{\text {*** }}$ & $164.81^{\text {** }}$ & $120.95^{* *}$ & $37.05^{*}$ & 6.02 \\
\hline 15 & $\mathbf{A S}$ & 0.37 & $102.19^{* *}$ & $129.83^{* *}$ & $94.95^{* *}$ & $24.07^{*}$ & 4.08 \\
\hline 16 & $\mathbf{S S}^{-1}$ & 1.83 & $1.07^{*}$ & $1.31^{*}$ & 1.01 & 0.18 & 0.69 \\
\hline 17 & RV & 0.18 & $115.84^{* *}$ & $255.57^{* *}$ & $70.25^{* *}$ & $79.98^{* *}$ & 0.39 \\
\hline 18 & $\mathbf{R L}$ & 0.36 & $38.35^{* *}$ & $62.49^{* *}$ & $31.21^{* *}$ & $5.93^{* *}$ & 0.48 \\
\hline 19 & RG & 0.11 & $2.54^{* *}$ & $3.99^{* *}$ & $2.12^{* *}$ & 0.29 & 0.09 \\
\hline 20 & TSW & 1.25 & $2.78^{* *}$ & $3.82^{* *}$ & $2.49^{* *}$ & 0.51 & 1.28 \\
\hline 21 & BY & 151560.50 & $183130.29^{* *}$ & $197067.52^{* *}$ & $177778.97^{* *}$ & 208530.50 & 79116.11 \\
\hline 22 & HI & 0.04 & $24.00^{* *}$ & $22.51^{* *}$ & $25.16^{* *}$ & 0.19 & 3.55 \\
\hline 23 & OC & 0.06 & $0.23^{*}$ & $0.34^{* *}$ & 0.18 & $0.63^{*}$ & 0.14 \\
\hline 24 & DME & 0.38 & $17.79^{* *}$ & $17.49^{* *}$ & $18.38^{* *}$ & 0.31 & 2.22 \\
\hline 25 & GYP $^{-1}$ & 0.51 & $20.14^{* *}$ & $19.96^{* *}$ & $20.71^{* *}$ & 1.75 & 5.38 \\
\hline
\end{tabular}


Table.2 Mean, range and coefficient of variance for 25 characters in Indian mustard genotypes under rainfed condition

\begin{tabular}{|c|c|c|c|c|c|c|c|c|c|c|c|c|}
\hline \multirow[t]{3}{*}{ No. } & \multirow[t]{3}{*}{ Characters } & \multirow[t]{3}{*}{ Mean } & \multicolumn{9}{|c|}{ Range } & \multirow[t]{3}{*}{$\mathbf{C V}$} \\
\hline & & & \multicolumn{3}{|c|}{ All Genotypes } & \multicolumn{3}{|c|}{ Basal Branching Genotypes (BB) } & \multicolumn{3}{|c|}{ Non -Basal Branching Genotypes (NBB) } & \\
\hline & & & Maximum & Max.-min & Minimum & Maximum & Max.-min & Minimum & Maximum & Max.-min & Minimum & \\
\hline 1 & DFFO & $39.11 \pm 0.96$ & RH-0116 & $42.67-35.67$ & R.Suphlam & Divya & $41.67-35.67$ & R.Suphlam & RH-0116 & $42.67-36.00$ & $\begin{array}{l}\text { PusaMahak, } \\
\text { RH-8701, } \\
\text { RH-8819 }\end{array}$ & 4.26 \\
\hline 2 & DFF & $99.12 \pm 0.70$ & $\begin{array}{l}\text { RH-0116, } \\
\text { Kanti, PM- } \\
25 \text {, } \\
\text { Divya }\end{array}$ & $103.00-95.00$ & $\begin{array}{l}\text { R.Suphlam, } \\
\text { PusaMahak }\end{array}$ & Divya & $\begin{array}{l}103.00- \\
95.00\end{array}$ & R.Suphlam & $\begin{array}{l}\text { RH0116, } \\
\text { Kanti, } \\
\text { PM-25 }\end{array}$ & $\begin{array}{l}103.00- \\
95.00\end{array}$ & PusaMahak & 1.23 \\
\hline 3 & DPM & $128.68 \pm 1.42$ & Kanti & $\begin{array}{l}133.00- \\
123.33\end{array}$ & R.Suphlam & Divya & $\begin{array}{l}132.00- \\
123.33\end{array}$ & R.Suphlam & KANTI & $\begin{array}{l}133.00- \\
123.67\end{array}$ & PusaMahak & 1.92 \\
\hline 4 & $\mathbf{P B P}^{-1}$ & $8.29 \pm 0.31$ & PusaMahak & $13.20-5.07$ & TM-2 & R.Suphlam & $12.80-5.07$ & TM-2 & PusaMahak & $13.20-5.33$ & Kanti & 6.49 \\
\hline 5 & $\mathbf{S B P}^{-1}$ & $12.01 \pm 0.66$ & PusaMahak & $26.12-3.90$ & TM-2 & R.Suphlam & $25.94-3.90$ & TM-2 & PusaMahak & $26.12-4.21$ & Kanti & 9.47 \\
\hline 6 & NS & $473.23 \pm 23.17$ & PusaMahak & $\begin{array}{l}1081.45- \\
199.79\end{array}$ & Kanti & R.Suphlam & $\begin{array}{l}999.63- \\
224.50\end{array}$ & TM-2 & PusaMahak & $\begin{array}{l}1018.45- \\
199.79\end{array}$ & Kanti & 8.48 \\
\hline 7 & LS & $4.89 \pm 0.16$ & R.Suphlam & $6.33-4.09$ & Kanti & R.Suphlam & $6.33-4.24$ & Divya & PusaMahak & $5.79-4.09$ & Kanti & 5.55 \\
\hline 8 & SG & $4.43 \pm 0.22$ & R.Suphlam & $8.40-2.33$ & Kanti & R.Suphlam & $8.40-2.65$ & Divya & PusaMahak & $7.90-2.33$ & Kanti & 8.74 \\
\hline 9 & IL & $11.16 \pm 0.47$ & R.Suphlam & $17.08-7.73$ & Kanti & R.Suphlam & $17.08-8.36$ & Divya & PusaMahak & $15.16-7.73$ & Kanti & 7.25 \\
\hline 10 & HP & $175.45 \pm 6.97$ & R.Suphlam & $\begin{array}{l}243.73- \\
117.26\end{array}$ & Kanti & R.Suphlam & $\begin{array}{l}243.73- \\
140.53\end{array}$ & Divya & PusaMahak & $\begin{array}{l}214.53- \\
117.26\end{array}$ & Kanti & 6.88 \\
\hline 11 & SPMA & $43.64 \pm 1.31$ & R.Suphlam & $65.67-34.93$ & Kanti & R.Suphlam & $65.67-36.33$ & TM-2 & PusaMahak & $56.33-34.93$ & Kanti & 5.20 \\
\hline 12 & HFPB & $44.42 \pm 2.56$ & Kanti & $93.60-9.93$ & R.Suphlam & TM-2 & $29.81-9.93$ & R.Suphlam & Kanti & $93.60-34.40$ & TPM-128 & 9.98 \\
\hline 13 & HFS & $113.38 \pm 4.97$ & Kanti & $178.18-35.67$ & R.Suphlam & Divya & $\begin{array}{l}149.77- \\
35.67\end{array}$ & R.Suphlam & Kanti & $\begin{array}{l}178.18- \\
76.19\end{array}$ & PusaMahak & 7.59 \\
\hline 14 & $\mathbf{A B}$ & $32.97 \pm 1.49$ & Kanti & $51.67-14.66$ & R.Suphlam & Divya & $43.66-14.66$ & R.Suphlam & Kanti & $51.67-20.66$ & PusaMahak & 7.86 \\
\hline 15 & $\mathbf{A S}$ & $28.77 \pm 1.43$ & Kanti & $42.00-13.33$ & R.Suphlam & Divya & $36.67-13.33$ & R.Suphlam & Kanti & $42.00-17.67$ & PusaMahak & 8.62 \\
\hline 16 & $\mathbf{S S}^{-1}$ & $11.052 \pm 0.48$ & R.Suphlam & $12.67-9.20$ & Kanti & R.Suphlam & $12.67-10.13$ & Divya & PusaMahak & $12.13-9.20$ & Kanti & 7.54 \\
\hline 17 & $\mathbf{R V}$ & $7.84 \pm 0.38$ & R.Suphlam & $35.23-1.71$ & Kanti & R.Suphlam & $35.23-2.72$ & Divya & PusaMahak & $24.02-1.71$ & Kanti & 8.46 \\
\hline 18 & RL & $12.08 \pm 0.40$ & R.Suphlam & $24.56-6.51$ & Kanti & R.Suphlam & $24.56-7.94$ & Divya & PusaMahak & $19.83-6.51$ & Kanti & 5.76 \\
\hline 19 & RG & $3.04 \pm 0.18$ & R.Suphlam & $5.97-1.71$ & Kanti & R.Suphlam & $5.97-2.01$ & Divya & NDRE-7 & $5.06-1.71$ & Kanti & 10.06 \\
\hline 20 & TSW & $5.25 \pm 0.65$ & R.Suphlam & $7.67-3.57$ & Kanti & R.Suphlam & $7.56-3.90$ & Divya & PusaMahak & $7.56-3.57$ & Kanti & 21.58 \\
\hline 21 & BY & $1966.2 \pm 162.39$ & R.Suphlam & $\begin{array}{l}2416.67- \\
1416.67\end{array}$ & RH-0116 & R.Suphlam & $\begin{array}{l}2416.67- \\
1500.00\end{array}$ & TM-2 & NDRE-7 & $\begin{array}{l}2366.67- \\
1416.67\end{array}$ & RH-0116 & 14.31 \\
\hline 22 & HI & $18.40 \pm 1.14$ & PusaMahak & $23.85-12.07$ & Kanti & R.Suphlam & $22.00-13.77$ & TM-2 & PusaMahak & $23.85-12.07$ & Kanti & 10.75 \\
\hline 23 & OC & $39.01 \pm 0.21$ & KMR10-1 & $39.57-38.36$ & Divya & R.Suphlam & $39.41-38.36$ & Divya & KMR10-1 & $39.57-38.47$ & BAUM-08-56 & 0.95 \\
\hline 24 & DME & $14.35 \pm 0.93$ & PusaMahak & $19.34-9.08$ & Kanti & R.Suphlam & $17.90-10.47$ & TM-2 & PusaMahak & $19.34-9.08$ & Kanti & 11.23 \\
\hline 25 & GYP $^{-1}$ & $24.77 \pm 1.34$ & R.Suphlam & 29.33-18.97 & Kanti & R.Suphlam & $29.33-20.90$ & Divya & PusaMahak & $28.57-18.97$ & Kanti & 9.36 \\
\hline
\end{tabular}


Table.3 Mean performance of 50 genotypes of for 25 characters Indian mustard genotypes under rainfed condition

\begin{tabular}{|c|c|c|c|c|c|c|c|c|c|c|c|c|c|c|}
\hline No & Genotypes & DFFO & DFF & DPM & PBP $^{1}$ & SBP $^{1}$ & NS & LS & SG & IL & HP & SPMA & HFPB & HFS \\
\hline 1 & NDRE7 & 36.00 & $95.33 * *$ & $124.33 * *$ & $9.93 * *$ & $19.33^{*}$ & $679.93 * *$ & $5.53^{* * *}$ & $7.27^{* *}$ & $14.53^{* *}$ & $214.33^{* *}$ & $58.07^{* *}$ & $16.42 *$ & 106.91 \\
\hline 2 & RH0406 & 40.67 & 100.67 & 130.00 & 6.60 & 8.23 & 329.34 & 4.49 & 3.21 & 9.75 & 163.27 & 38.60 & $9.94 * *$ & 120.65 \\
\hline 3 & DRMRLEJ902 & 37.33 & $97.00^{* * *}$ & 126.67 & 9.33 & 14.62 & 518.45 & 5.14 & $5.51^{* *}$ & 12.41 & 191.27 & 47.40 & 40.99 & 102.58 \\
\hline 4 & RH0116 & 42.67 & 103.00 & 132.33 & 5.53 & 4.41 & 200.49 & 4.13 & 2.43 & 7.85 & 120.07 & 35.40 & 79.00 & 152.40 \\
\hline 5 & RAURD-212 & 39.00 & 99.00 & 129.00 & 8.53 & 11.78 & 456.13 & 5.00 & 4.27 & 11.66 & 179.80 & 43.73 & 42.42 & 107.71 \\
\hline 6 & PUSA TARAK (EJ9913) & 40.00 & 100.00 & 129.33 & 7.60 & 10.73 & 455.69 & 4.91 & 3.99 & 11.09 & 175.40 & 42.13 & 21.33 & 110.45 \\
\hline 7 & PUSA AGRANI (SEJ-2) & 40.67 & 100.33 & 130.00 & 7.47 & 8.24 & 351.95 & 4.65 & 3.37 & 9.85 & 166.80 & 40.07 & 55.70 & 119.70 \\
\hline 8 & PM25 & 41.67 & 103.00 & 132.33 & 5.67 & 4.89 & 224.49 & 4.18 & 2.56 & 8.11 & 121.10 & 36.33 & 73.53 & 151.52 \\
\hline 9 & BAUM08-57 & 36.33 & 99.00 & 128.00 & 8.67 & 12.91 & 442.07 & 5.03 & 4.80 & 11.88 & 182.33 & 44.93 & 39.84 & 104.83 \\
\hline$\overline{10}$ & PUSA MAHAK (JD-6) & 36.00 & $95.00^{* * *}$ & $123.67 * *$ & $13.20^{* *}$ & $26.12^{* * *}$ & $1081.45 * *$ & $5.79 * *$ & $7.90^{* *}$ & $15.16^{* *}$ & $214.53^{* *}$ & $56.33 * *$ & 57.60 & 76.19 \\
\hline 11 & PKRS-28 & 38.33 & 99.00 & 128.33 & 8.87 & 14.36 & 519.13 & 5.02 & 4.76 & 11.85 & 181.87 & 44.53 & 22.33 & 106.90 \\
\hline 12 & NRCDR-2 & 36.00 & 96.00 *** & 125.00 & $10.80^{* * *}$ & 21.18 ** & 851.49 ** & 5.44 & $7.83 * *$ & $13.65 * *$ & $205.53 *$ & 52.53 ** & 38.70 & 117.65 \\
\hline 13 & PM-27 & 39.33 & 99.00 & 129.00 & 8.40 & 11.64 & 451.56 & 4.99 & 4.20 & 11.51 & 178.27 & 43.27 & 42.57 & 108.14 \\
\hline 14 & PM-28 (NPJ-124) & 39.00 & 99.00 & 128.67 & 8.87 & 14.11 & 494.36 & 5.02 & 4.43 & 11.73 & 179.87 & 43.93 & 21.40 & 107.15 \\
\hline 15 & PANT RAI & 40.33 & 100.00 & 130.00 & 7.67 & 9.12 & 364.22 & 4.76 & 3.60 & 10.34 & 168.80 & 40.80 & 54.67 & 115.90 \\
\hline 16 & KRISHNA & 40.67 & 100.00 & 130.00 & 7.47 & 8.34 & 375.19 & 4.71 & 3.43 & 9.93 & 167.80 & 40.20 & 55.00 & 118.99 \\
\hline 17 & TM 151 & 37.00 & $96.00 * *$ & 126.00 & 9.67 ** & $17.33^{* *}$ & $636.47^{* * *}$ & 5.21 & $6.00^{* * *}$ & 12.71 & 192.20 & $50.07 * *$ & 43.67 & 98.68 \\
\hline 18 & DIVYA & 41.67 & 103.00 & 132.00 & 6.40 & 5.89 & 281.23 & 4.24 & 2.65 & 8.36 & 140.53 & 36.73 & 20.61 & 149.77 \\
\hline 19 & MAYA & 37.33 & $96.00^{* * *}$ & 126.00 & 9.40 & $15.77^{*}$ & $586.70^{* * *}$ & 5.20 & $6.00^{* * *}$ & 12.65 & 192.07 & $49.53 * *$ & 40.12 & 99.91 \\
\hline 20 & ROHINI & 41.00 & 101.33 & 131.00 & 6.53 & 6.42 & 277.03 & 4.44 & 2.87 & 9.08 & 148.93 & 36.80 & 64.53 & 141.66 \\
\hline 21 & DRMR150-35 & 36.67 & $96.00^{* * *}$ & 126.00 & $9.80^{*}$ & $18.12^{* * *}$ & $706.00^{* * *}$ & 5.25 & $6.13^{* * *}$ & 12.95 & 196.40 & $50.47^{* * *}$ & 41.05 & 97.15 \\
\hline 22 & BASANTI & 38.00 & 98.00 & 127.67 & 8.73 & 13.07 & 489.26 & 5.05 & 4.85 & 12.00 & 187.60 & 45.73 & 37.80 & 104.13 \\
\hline 23 & TM-4 & 40.67 & 101.00 & 130.33 & 7.13 & 7.75 & 303.31 & 4.54 & 3.15 & 9.60 & 163.73 & 37.47 & 59.50 & 121.99 \\
\hline 24 & RH-30 & 40.00 & 100.00 & 129.33 & 8.13 & 10.43 & 423.60 & 4.87 & 3.86 & 11.01 & 173.33 & 42.07 & 43.77 & 110.99 \\
\hline 25 & KRANTI & 40.00 & 100.00 & 129.67 & 8.07 & 10.04 & 452.93 & 4.87 & 3.76 & 10.95 & 173.20 & 41.60 & 46.83 & 111.15 \\
\hline 26 & TM215 & 40.33 & 100.00 & 129.67 & 8.27 & 12.57 & 517.85 & 4.83 & 3.73 & 10.74 & 171.67 & 41.47 & 29.80 & 112.48 \\
\hline 27 & RGN-13 & 40.67 & 101.00 & 130.33 & 6.80 & 8.90 & 331.67 & 4.48 & 3.12 & 9.47 & 160.33 & 37.27 & 19.37 & 122.31 \\
\hline 28 & BAUM08-56 & 37.33 & $97.00^{*}$ & 127.00 & $9.87 * *$ & $17.61^{* * *}$ & $621.41 * *$ & 5.09 & 5.07 & 12.35 & 190.20 & 46.47 & 25.60 & 103.59 \\
\hline 29 & SHIVANI & 37.67 & 98.00 & 127.67 & 8.87 & 13.17 & 504.64 & 5.06 & 4.85 & 12.21 & 187.93 & 46.00 & 37.03 & 104.11 \\
\hline 30 & TM-2 & 41.33 & 102.00 & 131.67 & 5.07 & 3.90 & 224.50 & 4.32 & 2.77 & 8.37 & 142.53 & 36.33 & 29.81 & 147.87 \\
\hline 31 & RH0701 & 41.00 & 101.00 & 131.00 & 6.73 & 6.89 & 311.85 & 4.44 & 2.99 & 9.42 & 155.93 & 36.87 & 61.73 & 131.75 \\
\hline 32 & RAURD (E)-1002 & 41.00 & 102.00 & 131.00 & 6.20 & 5.69 & 305.50 & 4.42 & 2.85 & 8.65 & 147.87 & 37.33 & 65.07 & 145.32 \\
\hline 33 & KANTI & 42.00 & 103.00 & 133.00 & 5.33 & 4.21 & 199.79 & 4.09 & 2.33 & 7.73 & 117.26 & 34.93 & 93.60 & 178.18 \\
\hline 34 & NDRE-4 & 40.67 & 100.67 & 130.00 & 7.40 & 8.17 & 358.75 & 4.56 & 3.29 & 9.79 & 166.13 & 38.73 & 55.73 & 120.63 \\
\hline 35 & RH8701 & 36.00 & $96.00^{* * *}$ & 126.00 & $10.27 * *$ & $18.98 * *$ & $704.76^{* * *}$ & 5.39 & $6.60^{* * *}$ & 12.97 & 199.87 & $51.20^{* * *}$ & 72.96 & 89.35 \\
\hline 36 & PUSA BAHAR & 40.33 & 100.00 & 129.67 & 7.80 & 9.65 & 379.34 & 4.82 & 3.68 & 10.45 & 169.27 & 41.33 & 49.47 & 113.77 \\
\hline 37 & RAURD214 & 41.00 & 101.00 & 130.67 & 6.87 & 7.13 & 314.67 & 4.48 & 3.07 & 9.43 & 159.60 & 37.20 & 60.47 & 127.59 \\
\hline 38 & RGN-48 & 41.00 & 101.00 & 131.00 & 6.60 & 6.52 & 313.35 & 4.44 & 2.92 & 9.39 & 154.80 & 36.80 & 64.50 & 135.21 \\
\hline 39 & RH-8814 & 37.33 & $97.00^{*}$ & 127.00 & 9.27 & 14.50 & 517.71 & 5.16 & $5.39 * *$ & 12.41 & 191.13 & 47.07 & 45.91 & 102.61 \\
\hline 40 & RH-0819 & 36.00 & $96.00^{* * *}$ & 125.33 & $10.30^{* * *}$ & $19.46^{* * *}$ & $769.61^{* * *}$ & 5.39 & $6.93 * *$ & $13.32^{*}$ & $204.98^{*}$ & $52.33^{* * *}$ & 59.67 & 87.82 \\
\hline 41 & RAURD-78 & 37.67 & 97.67 & 127.00 & 8.80 & 13.19 & 527.72 & 5.07 & 4.91 & 12.26 & 189.53 & 46.47 & 35.34 & 104.00 \\
\hline 42 & TPM-128 & 37.33 & $97.00^{*}$ & 126.67 & 9.60 & $16.93^{* * *}$ & $657.64 * *$ & 5.18 & $5.59^{* * *}$ & 12.59 & 191.87 & 47.40 & 34.40 & 101.50 \\
\hline 43 & RH8812 & 40.00 & 100.00 & 129.33 & 8.33 & 10.98 & 418.79 & 4.91 & 4.07 & 11.19 & 176.73 & 42.20 & 43.40 & 109.77 \\
\hline 44 & KMR10-1 & 37.33 & $97.00 *$ & 127.00 & 9.07 & 13.86 & 472.33 & 5.09 & $5.13^{* * *}$ & 12.37 & 190.27 & 46.53 & 65.93 & 103.31 \\
\hline 45 & KM R10-2 & 38.33 & 98.67 & 127.67 & 9.33 & $15.76^{*}$ & $609.63 * *$ & 5.04 & 4.80 & 11.91 & 185.67 & 45.27 & $17.60^{*}$ & 104.73 \\
\hline 46 & RAURD (E)-1001 & 39.67 & 99.33 & 129.00 & 8.27 & 10.70 & 400.98 & 4.94 & 4.18 & 11.35 & 178.67 & 42.80 & 42.87 & 109.67 \\
\hline 47 & TPM-1 & 40.33 & 100.00 & 130.00 & 7.73 & 9.39 & 416.95 & 4.81 & 3.67 & 10.39 & 168.27 & 40.93 & 50.80 & 115.52 \\
\hline 48 & VARUNA & 38.67 & 99.00 & 128.33 & 8.73 & 13.10 & 455.37 & 5.02 & 4.47 & 11.79 & 181.67 & 44.07 & 25.93 & 80.30 \\
\hline 49 & PUSA BOLD & 40.33 & 100.00 & 130.00 & 7.60 & 8.46 & 374.70 & 4.75 & 3.66 & 10.01 & 167.60 & 40.53 & 54.73 & 85.49 \\
\hline \multirow{4}{*}{50} & RAJENDRA SUPHLAM & $35.67^{*}$ & $95.00 * *$ & $123.33^{* * *}$ & $12.80^{* * *}$ & $25.94 * *$ & 999.63** & $6.33 * *$ & 8.40 ** & $17.08^{* *}$ & $243.73 * *$ & $65.67 * *$ & $9.93 * *$ & $68.94 *$ \\
\hline & Mean & 39.11 & 99.12 & 128.68 & 8.29 & 12.01 & 473.23 & 4.89 & 4.43 & 11.16 & 175.45 & 43.64 & 44.42 & 113.38 \\
\hline & S.E. & 0.96 & 0.70 & 1.42 & 0.31 & 0.66 & 23.17 & 0.16 & 0.22 & 0.47 & 6.97 & 1.31 & 2.56 & 4.96 \\
\hline & C.D. 5\% & 2.70 & 1.98 & 3.99 & 0.87 & 1.84 & 65.03 & 0.44 & 0.63 & 1.31 & 19.55 & 3.68 & 7.18 & 13.96 \\
\hline
\end{tabular}




\begin{tabular}{|c|c|c|c|c|c|c|c|c|c|c|c|c|c|c|}
\hline No. & Genotypes & Character & $\mathbf{A B}$ & AS & $\mathrm{SS}^{-1}$ & RV & $\mathbf{R L}$ & RG & TSW & BY & HII & $\mathbf{O C}$ & DME & GYP $^{-1}$ \\
\hline 1 & NDRE7 & & 23.00 & 18.67 & 11.80 & $21.81 * *$ & 18.91 ** & $5.06 *$ & $7.39^{*}$ & 2366.67 & $23.14 * *$ & 38.95 & $18.62^{* *}$ & 28.33 \\
\hline 2 & RH0406 & & 36.00 & 32.67 & 10.73 & 4.20 & 9.77 & 2.47 & 4.66 & 1800.00 & 16.04 & 38.78 & 12.34 & 22.60 \\
\hline 3 & DRMRLEJ902 & & 29.67 & 24.33 & 11.53 & $9.42^{* * *}$ & $13.89 * *$ & 3.44 & 5.78 & 2200.00 & 20.40 & 39.22 & 16.10 & 26.83 \\
\hline 4 & RH0116 & & $45.67 * *$ & $38.07 * *$ & 10.07 & 2.49 & 6.75 & 1.76 & 3.72 & 1416.67 & 12.47 & 38.99 & 9.40 & 20.00 \\
\hline 5 & RAURD-212 & & 32.00 & 28.00 & 11.20 & 6.47 & 11.65 & 2.91 & 5.27 & 2013.33 & 20.15 & 38.96 & 15.61 & 25.63 \\
\hline 6 & PUSA TARAK (EJ9913) & & 32.33 & 29.00 & 11.13 & 5.52 & 11.34 & 2.78 & 5.12 & 1946.67 & 18.48 & 38.75 & 14.32 & 25.10 \\
\hline 7 & PUSA AGRANI (SEJ-2) & & 35.33 & 32.67 & 10.80 & 4.57 & 10.47 & 2.53 & 4.77 & 1983.33 & 15.83 & 38.70 & 12.18 & 23.30 \\
\hline 8 & PM25 & & $44.33 * *$ & $37.67^{* * *}$ & 10.13 & 2.70 & 7.67 & 1.95 & 3.90 & 1508.33 & 13.09 & 38.98 & 9.89 & 20.70 \\
\hline 9 & BAUM08-57 & & 31.33 & 26.67 & 11.27 & 7.35 & 11.93 & 3.09 & 5.47 & 2083.33 & 19.93 & 39.42 & 15.57 & 26.07 \\
\hline 10 & PUSA MAHAK (JD-6) & & 20.67 & 17.67 & 12.13 & $24.02 * *$ & $19.83 * *$ & $5.02 * *$ & $7.56 *$ & 2166.67 & $23.85 * *$ & 38.62 & $19.34 * *$ & $28.57^{*}$ \\
\hline 11 & PKRS-28 & & 31.67 & 26.67 & 11.20 & 7.09 & 11.76 & 3.07 & 5.46 & 1966.67 & 19.43 & 38.83 & 15.14 & 25.97 \\
\hline 12 & NRCDR-2 & & 23.00 & 20.00 & 11.80 & $18.94^{* * *}$ & $17.65^{* *}$ & $4.75 * *$ & 6.88 & 2213.33 & $21.92^{*}$ & 39.05 & $17.54^{*}$ & 28.07 \\
\hline 13 & PM-27 & & 32.00 & 28.00 & 11.20 & 5.95 & 11.57 & 2.90 & 5.24 & 2173.33 & 18.96 & 39.13 & 14.72 & 25.43 \\
\hline 14 & PM-28 (NPJ-124) & & 31.67 & 27.67 & 11.20 & 6.65 & 11.65 & 2.55 & 5.29 & 2363.33 & 19.34 & 38.69 & 15.04 & 25.67 \\
\hline 15 & PANT RAI & & 34.33 & 31.00 & 10.87 & 4.67 & 11.05 & 2.61 & 4.95 & 1850.00 & 17.52 & 38.99 & 13.48 & 24.13 \\
\hline 16 & KRISHNA & & 35.00 & 32.33 & 10.80 & 4.62 & 10.71 & 2.53 & 4.78 & 1966.67 & 16.30 & 39.10 & 12.55 & 23.50 \\
\hline 17 & TM 151 & & 28.00 & 23.00 & 11.60 & $12.15^{* * *}$ & $16.35 * *$ & $3.92^{* * *}$ & 6.07 & 2233.33 & 21.18 & 38.97 & 16.79 & 27.33 \\
\hline 18 & DIVYA & & $43.67 * *$ & $36.67^{* * *}$ & 10.13 & 2.73 & 7.94 & 2.01 & 3.90 & 1863.33 & 14.63 & 38.36 & 11.09 & 20.90 \\
\hline 19 & MAYA & & 28.33 & 23.33 & 11.53 & $11.61 * *$ & $15.23 * *$ & $3.76 * *$ & 5.95 & 2233.33 & 20.78 & 39.24 & 16.50 & 27.50 \\
\hline 20 & ROHINI & & $40.67 * *$ & $36.00^{* * *}$ & 10.40 & 3.46 & 8.17 & 2.20 & 4.12 & 1630.00 & 15.23 & 38.72 & 11.62 & 21.73 \\
\hline 21 & DRMR150-35 & & 26.33 & 22.67 & 11.63 & $14.01 * *$ & $16.73 * *$ & $4.31^{* *}$ & 6.19 & 2133.33 & $22.59^{*}$ & 39.23 & $17.93 * *$ & 27.63 \\
\hline 22 & BASANTI & & 30.67 & 26.00 & 11.33 & 7.57 & 12.05 & 3.11 & 5.64 & 2000.00 & 19.40 & 38.64 & 15.19 & 26.37 \\
\hline 23 & TM-4 & & 36.33 & 33.67 & 10.64 & 4.06 & 9.53 & 2.39 & 4.60 & 1733.33 & 22.17 & 39.10 & $17.02 *$ & 22.40 \\
\hline 24 & RH-30 & & 32.33 & 29.33 & 11.07 & 5.41 & 11.30 & 2.79 & 5.06 & 1866.67 & 18.46 & 39.03 & 14.31 & 24.73 \\
\hline 25 & KRANTI & & 32.67 & 30.00 & 11.07 & 5.38 & 11.29 & 2.71 & 5.04 & 1900.00 & 18.20 & 38.90 & 14.08 & 24.60 \\
\hline$\frac{26}{26}$ & TM215 & & 32.67 & 30.33 & 11.00 & 5.11 & 11.21 & 2.67 & 5.03 & 1900.00 & 18.15 & 39.30 & 14.00 & 24.50 \\
\hline 27 & RGN-13 & & 36.67 & $34.00^{* * *}$ & 10.47 & 4.03 & 9.35 & 2.36 & 4.45 & 1950.00 & 15.93 & 38.62 & 12.23 & 22.30 \\
\hline 28 & BAUM08-56 & & 30.33 & 25.00 & 11.33 & 8.74 & $13.15 * *$ & 3.29 & 5.72 & 2141.67 & 20.11 & 38.47 & 15.85 & 26.67 \\
\hline 29 & SHIVANI & & 30.33 & 26.00 & 11.33 & 7.71 & 12.19 & 3.13 & 5.64 & 1780.00 & 20.25 & 39.08 & 15.85 & 26.43 \\
\hline 30 & TM-2 & & $42.00 * *$ & 36.00 & 10.27 & 2.80 & 8.06 & 2.04 & 3.94 & 1500.00 & 13.77 & 39.28 & 10.47 & 21.27 \\
\hline 31 & RH0701 & & $39.00 * *$ & $34.33^{* * *}$ & 10.53 & 3.83 & 8.88 & 2.27 & 4.16 & 1683.33 & 15.79 & 39.43 & 12.05 & 21.90 \\
\hline 32 & RAURD (E)-1002 & & $41.67 * *$ & 36.00 & 10.27 & 3.37 & 8.09 & 2.11 & 4.12 & 1566.67 & 14.41 & 39.22 & 11.01 & 21.40 \\
\hline 33 & KANTI & & $51.67 * *$ & $42.00^{* * *}$ & 9.20 & 1.71 & 6.51 & 1.71 & 3.57 & 1600.00 & 12.07 & 39.05 & 9.08 & 18.97 \\
\hline 34 & NDRE-4 & & 36.00 & 32.67 & 10.80 & 4.36 & 9.95 & 2.52 & 4.72 & 1800.00 & 16.09 & 39.22 & 12.38 & 23.10 \\
\hline 35 & RH8701 & & 25.33 & 22.33 & 11.67 & $14.14 * *$ & $16.93 * *$ & $4.59^{* *}$ & 6.44 & 2000.00 & 20.84 & 38.73 & 16.55 & 27.87 \\
\hline 36 & PUSA BAHAR & & 33.67 & 30.67 & 10.97 & 4.72 & 11.11 & 2.78 & 5.00 & 1880.00 & 17.91 & 39.24 & 13.82 & 24.40 \\
\hline 37 & RAURD214 & & $38.00^{*}$ & $34.33 * *$ & 10.53 & 4.03 & 8.94 & 2.34 & 4.23 & 1683.33 & 15.88 & 39.09 & 12.16 & 22.03 \\
\hline 38 & RGN-48 & & $39.33 * *$ & 35.33 & 10.47 & 3.48 & 8.55 & 2.23 & 4.13 & 1566.67 & 15.52 & 39.13 & 11.85 & 19.50 \\
\hline 39 & RH-8814 & & 29.67 & 24.33 & 11.47 & 9.25 & $13.77 * *$ & 3.35 & 5.77 & 2193.33 & 19.95 & 39.10 & 15.71 & 26.73 \\
\hline 40 & RH-0819 & & 24.33 & 22.33 & 11.73 & $14.89 * *$ & $17.57 * *$ & $4.63 * *$ & 6.73 & 2333.33 & 21.52 & 38.76 & $17.18^{*}$ & 28.00 \\
\hline 41 & RAURD-78 & & 30.33 & 25.33 & 11.33 & 8.45 & 13.15 & 3.18 & 5.69 & 2133.33 & 20.52 & 38.70 & 16.15 & 26.57 \\
\hline$\frac{71}{42}$ & TPM-128 & & 29.67 & 23.67 & 11.53 & $9.57 * *$ & $14.55 * *$ & $3.59 *$ & 5.87 & 2216.67 & 19.95 & 39.42 & 15.76 & 27.33 \\
\hline 43 & RH8812 & & 32.33 & 29.00 & 11.13 & 5.57 & 11.49 & 2.81 & 5.17 & 1983.33 & 18.62 & 39.43 & 14.39 & 25.20 \\
\hline 44 & KMR10-1 & & 30.00 & 24.33 & 11.33 & $8.97 * *$ & $13.55 * *$ & 3.33 & 5.73 & 2163.33 & 19.72 & 39.57 & 15.53 & 26.70 \\
\hline 45 & KM R10-2 & & 31.33 & 26.33 & 11.27 & 7.36 & 11.96 & 3.11 & 5.53 & 2100.00 & 19.42 & 39.21 & 15.22 & 26.13 \\
\hline 46 & RAURD (E)-1001 & & 32.00 & 28.67 & 11.13 & 5.81 & 11.50 & 2.89 & 5.18 & 2000.00 & 18.92 & 38.84 & 14.68 & 25.33 \\
\hline 47 & TPM-1 & & 34.00 & 31.00 & 10.87 & 4.71 & 11.07 & 2.67 & 5.00 & 1863.33 & 17.17 & 38.76 & 13.20 & 24.23 \\
\hline 48 & VARUNA & & 31.67 & 27.67 & 11.20 & 6.67 & 11.71 & 3.07 & 5.37 & 2063.33 & 19.57 & 39.08 & 15.26 & 25.70 \\
\hline 49 & PUSA BOLD & & 34.67 & 31.67 & 10.80 & 4.64 & 10.95 & 2.57 & 4.89 & 2180.00 & 16.44 & 39.06 & 12.65 & 23.93 \\
\hline \multirow[t]{4}{*}{50} & RAJENDRA SUPHLAM & & 14.67 & 13.33 & $12.67 *$ & $35.23 * *$ & 24.56 *** & $5.97 * *$ & $7.67 * *$ & 2416.67 & $22.00 *$ & 39.41 & $17.90 * *$ & $29.33^{*}:$ \\
\hline & Mean & & 32.97 & 28.77 & 11.05 & 7.84 & 12.08 & 3.04 & 5.25 & 1966.20 & 18.40 & 39.01 & 14.35 & 24.77 \\
\hline & S.E. & & 1.49 & 1.43 & 0.48 & 0.38 & 0.40 & 0.18 & 0.65 & 162.39 & 1.14 & 0.21 & 0.93 & 1.34 \\
\hline & C.D. $5 \%$ & & 4.19 & 4.02 & 1.35 & 1.07 & 1.13 & 0.49 & 1.84 & 455.75 & 3.21 & 0.60 & 2.61 & 3.76 \\
\hline
\end{tabular}

*Superior to Varuna (check) 
Table.4 Characterization of Basal Branching Indian mustard Genotypes for Morphological Attributes

\begin{tabular}{|c|c|c|c|c|c|c|c|c|c|c|c|}
\hline \multirow[t]{3}{*}{ S.N } & \multirow[t]{3}{*}{ GENOTYPES } & \multicolumn{2}{|c|}{ PRIMARY BRANCHES } & \multicolumn{2}{|c|}{$\begin{array}{l}\text { SECONDARY } \\
\text { BRANCHES }\end{array}$} & \multicolumn{2}{|c|}{ NO. OF SILIQUA } & \multicolumn{2}{|c|}{ INTERNODE LENGTH } & \multicolumn{2}{|c|}{1000 SEED WEIGHT } \\
\hline & & BB & NBB & BB & NBB & BB & NBB & BB & NBB & BB & NBB \\
\hline & & MEAN & MEAN & MEAN & MEAN & MEAN & MEAN & MEAN & MEAN & MEAN & MEAN \\
\hline 1 & NDRE7 & 4.40 & 5.53 & $12.12^{*}$ & $7.21^{*}$ & $217.27^{*}$ & $462.66^{*}$ & $6.18^{*}$ & $8.35^{*}$ & 4.11 & 3.28 \\
\hline 2 & RHO406 & 2.47 & 4.13 & 4.57 & 3.66 & 67.32 & 262.72 & 9.67 & 12.41 & $5.26^{*}$ & 4.16 \\
\hline 3 & $\begin{array}{l}\text { PUSA } \\
\text { TARAK } \\
\text { (EJ9913) }\end{array}$ & 2.93 & 4.67 & 6.33 & 4.40 & 98.53 & 306.25 & 8.89 & 10.95 & $5.58^{*}$ & $4.60^{*}$ \\
\hline 4 & PKRS-28 & 3.53 & 5.33 & 8.02 & $6.34^{*}$ & $135.14^{*}$ & 406.69 & 7.33 & 9.79 & $5.97^{*}$ & $5.18^{*}$ \\
\hline 5 & $\begin{array}{l}\text { PM-28 (NPJ- } \\
124)\end{array}$ & 3.60 & 5.27 & 8.13 & $5.98^{*}$ & 125.80 & 391.39 & 7.81 & 9.93 & $5.96^{*}$ & $5.17^{*}$ \\
\hline 6 & DIVYA & 2.40 & 4.00 & 3.63 & 2.26 & 63.31 & 235.15 & 11.60 & 12.71 & $5.12^{*}$ & 4.12 \\
\hline 7 & TM215 & 3.53 & 4.73 & 7.66 & 4.92 & 122.67 & 343.42 & 8.41 & 10.74 & $5.75^{*}$ & $4.77^{*}$ \\
\hline 8 & RGN-13 & 2.67 & 4.13 & 5.25 & 3.65 & 86.79 & 266.12 & 9.09 & 11.35 & $5.52^{*}$ & $4.23^{*}$ \\
\hline 9 & BAUM08-56 & 4.33 & 5.53 & $10.49^{*}$ & $7.12^{*}$ & $178.26^{*}$ & $439.10^{*}$ & $6.48^{*}$ & 8.65 & $6.04^{*}$ & $5.69^{*}$ \\
\hline 10 & TM-2 & 1.13 & 3.93 & 1.65 & 2.25 & 26.32 & 215.87 & 11.69 & 13.32 & 4.44 & 3.94 \\
\hline 11 & KM R10-2 & 3.93 & 5.40 & $9.34^{*}$ & $6.42^{*}$ & $152.64^{*}$ & 418.64 & $6.63^{*}$ & 9.60 & $5.99^{*}$ & $5.47^{*}$ \\
\hline 12 & VARUNA & 3.53 & 5.20 & 7.68 & 5.42 & 124.54 & 330.83 & 4.83 & 6.96 & 2.88 & 2.49 \\
\hline 13 & $\begin{array}{l}\text { RAJENDRA } \\
\text { SUPLAM }\end{array}$ & $6.20^{*}$ & $6.60^{*}$ & $17.26^{*}$ & $8.68^{*}$ & $270.44^{*}$ & $738.33^{*}$ & $5.33^{*}$ & $8.36^{*}$ & $7.51^{*}$ & $6.73^{*}$ \\
\hline$\overline{\text { GM }}$ & & 3.43 & 4.96 & 7.85 & 5.25 & 128.39 & 370.55 & 7.99 & 10.24 & 5.39 & 4.60 \\
\hline SE & & 0.36 & 0.27 & 0.39 & 0.31 & 28.82 & 33.43 & 0.63 & 0.52 & 0.79 & 0.76 \\
\hline CD & & 1.07 & 0.86 & 1.15 & 0.24 & 84.63 & 89.54 & 1.84 & 1.62 & 1.72 & 1.68 \\
\hline $\mathbf{C V}$ & & 7.49 & 10.19 & 5.17 & 13.65 & 9.96 & 9.43 & 9.44 & 8.33 & 25.56 & 19.71 \\
\hline
\end{tabular}


Table.5 Glyph - Array arrangement of 50 basal and non-basal branching Indian mustard genotypes under Rainfed condition

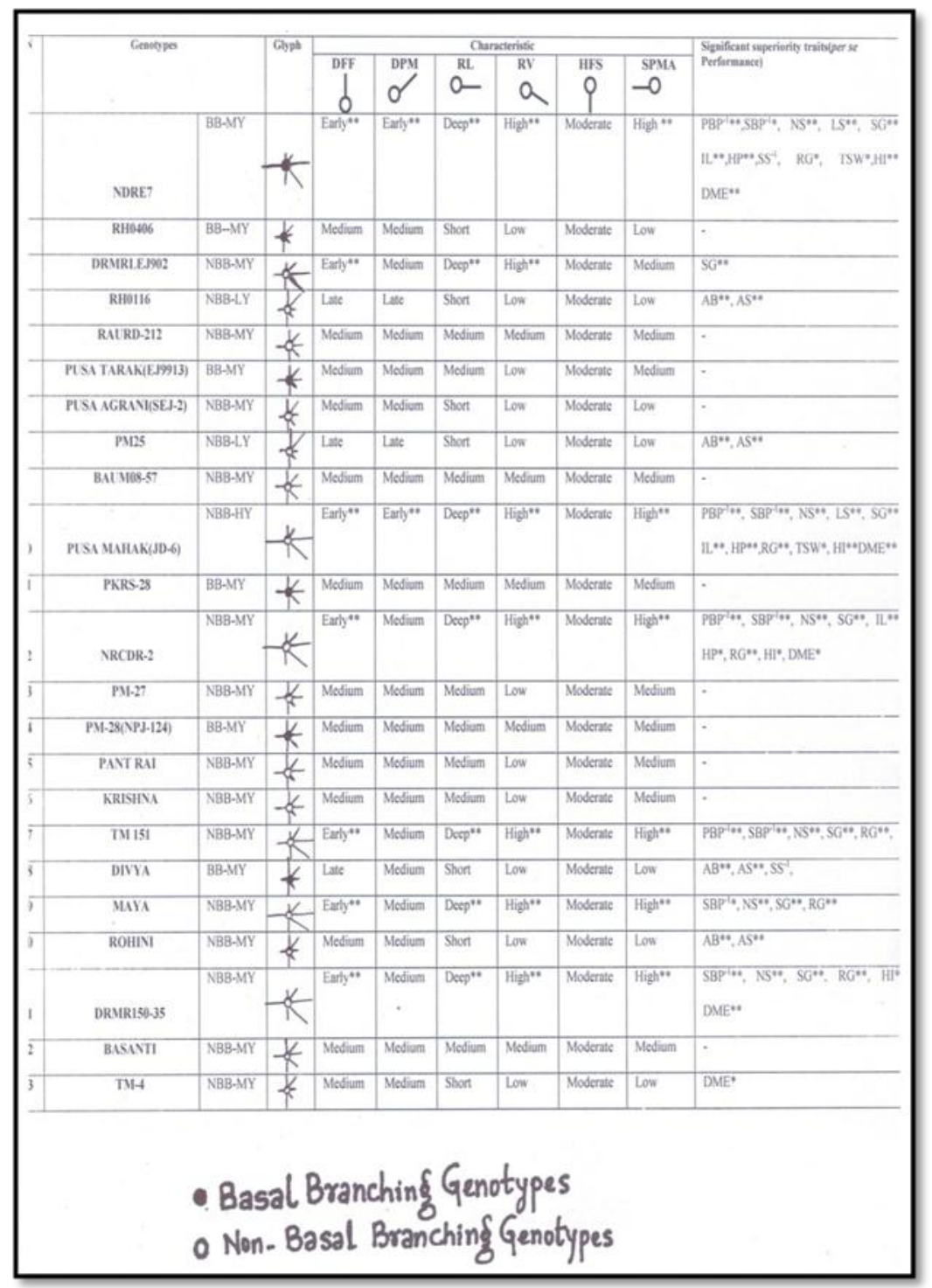

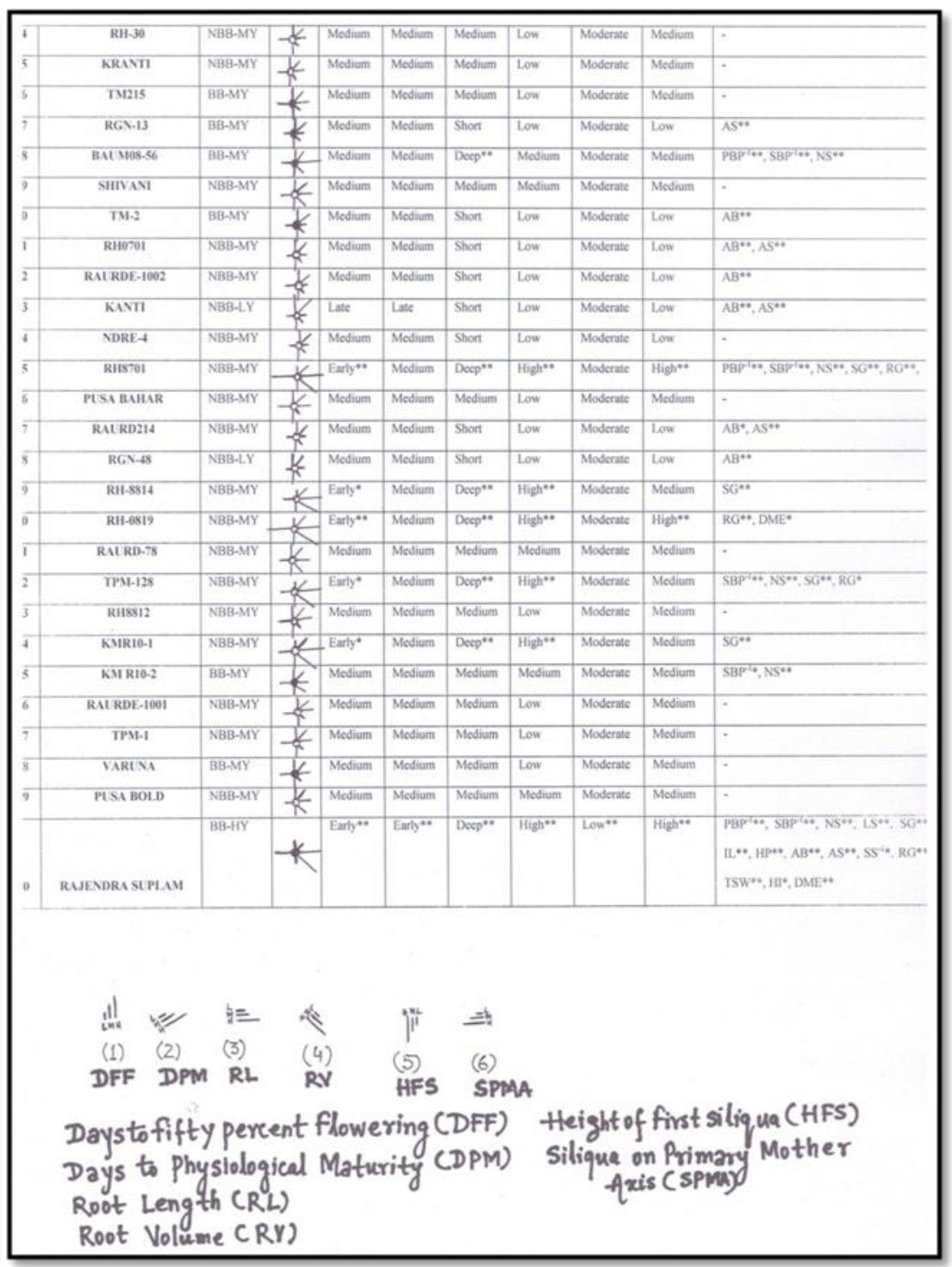


Table.6 Genetic parameters of 25 characters in Indian mustard genotypes

\begin{tabular}{|c|c|c|c|c|c|c|c|c|c|c|}
\hline No. & Characters & $\begin{array}{l}\text { Phenotypic } \\
\text { Variance } \\
\left(\sigma_{p}^{2}\right)\end{array}$ & $\begin{array}{l}\text { Genotypic } \\
\text { Variance } \\
\left(\sigma_{\mathrm{g}}^{2}\right)\end{array}$ & $\begin{array}{l}\text { Environmental } \\
\text { Variance } \\
\qquad\left(\sigma_{\mathrm{e}}^{2}\right)\end{array}$ & $\begin{array}{l}\text { Phenotypic } \\
\text { Coefficient } \\
\text { of Variance } \\
\text { (PCV) }\end{array}$ & $\begin{array}{l}\text { Genotypic } \\
\text { Coefficient } \\
\text { of Variance } \\
\text { (GCV) }\end{array}$ & $\begin{array}{l}\text { Environmental } \\
\text { Coefficient of } \\
\text { Variance } \\
\qquad \text { (ECV) }\end{array}$ & $\begin{array}{l}\text { Heritability } \\
\left(\mathbf{h}^{2} \mathrm{bs}\right)\end{array}$ & $\begin{array}{l}\text { Genetic } \\
\text { Advance }\end{array}$ & $\begin{array}{l}\text { Genetic } \\
\text { Advance } \\
\text { as \% of } \\
\text { Mean }\end{array}$ \\
\hline 1 & DFFO & 5.66 & 2.88 & 2.78 & 6.08 & 4.34 & 4.26 & 0.51 & 2.50 & 6.38 \\
\hline 2 & DFF & 6.04 & 4.56 & 1.49 & 2.48 & 2.15 & 1.23 & 0.75 & 3.82 & 3.85 \\
\hline 3 & DPM & 9.40 & 3.33 & 6.07 & 2.38 & 1.42 & 1.91 & 0.35 & 2.24 & 1.74 \\
\hline 4 & $\mathbf{P B P}^{-1}$ & 3.03 & 2.74 & 0.29 & 21.01 & 19.88 & 6.49 & 0.90 & 3.24 & 39.15 \\
\hline 5 & SBP $^{-1}$ & 28.81 & 27.52 & 1.29 & 44.70 & 43.68 & 9.47 & 0.96 & 10.56 & 87.94 \\
\hline 6 & NS & 37043.36 & 35432.35 & 1611 & 40.67 & 39.78 & 8.48 & 0.96 & 379.24 & 80.14 \\
\hline 7 & $\mathbf{L S}$ & 0.23 & 0.16 & 0.07 & 9.90 & 8.20 & 5.55 & 0.69 & 0.68 & 13.99 \\
\hline 8 & SG & 2.41 & 2.26 & 0.15 & 35.04 & 33.94 & 8.74 & 0.94 & 3.00 & 67.70 \\
\hline 9 & IL & 4.41 & 3.48 & 0.66 & 18.22 & 16.71 & 7.25 & 0.84 & 3.53 & 31.58 \\
\hline 10 & HP & 673.36 & 528.01 & 145.65 & 14.79 & 13.10 & 6.88 & 0.78 & 41.91 & 23.89 \\
\hline 11 & SPMA & 43.99 & 38.84 & 5.15 & 15.20 & 14.28 & 5.20 & 0.88 & 12.06 & 27.64 \\
\hline 12 & HFPB & 359.14 & 339.49 & 19.65 & 42.66 & 41.48 & 9.98 & 0.95 & 36.90 & 83.08 \\
\hline 13 & HFS & 482.45 & 408.22 & 74.23 & 19.37 & 17.82 & 7.60 & 0.85 & 38.29 & 33.77 \\
\hline 14 & AB & 47.80 & 41.09 & 6.71 & 20.97 & 19.44 & 7.86 & 0.86 & 12.24 & 37.14 \\
\hline 15 & $\mathbf{A S}$ & 38.16 & 32.01 & 6.15 & 21.47 & 19.67 & 8.62 & 0.84 & 10.68 & 37.11 \\
\hline 16 & $\mathbf{S S}^{-1}$ & 0.82 & 0.12 & 0.69 & 8.19 & 3.19 & 7.54 & 0.15 & 0.28 & 2.56 \\
\hline 17 & RV & 38.91 & 38.47 & 0.44 & 79.56 & 79.11 & 8.46 & 0.99 & 12.70 & 62.04 \\
\hline 18 & $\mathbf{R L}$ & 13.11 & 12.62 & 0.48 & 29.97 & 29.41 & 5.76 & 0.96 & 7.18 & 59.46 \\
\hline 19 & RG & 0.91 & 0.81 & 0.09 & 31.38 & 29.73 & 10.06 & 0.90 & 1.76 & 58.01 \\
\hline 20 & TSW & 1.78 & 0.50 & 1.28 & 25.42 & 13.44 & 21.58 & 0.28 & 0.77 & 14.64 \\
\hline 21 & BY & 113787.50 & 34671.39 & 79116.11 & 17.16 & 9.47 & 14.31 & 0.30 & 211.73 & 10.77 \\
\hline 22 & HI & 10.61 & 6.70 & 3.91 & 17.70 & 14.06 & 10.75 & 0.63 & 42.24 & 23.02 \\
\hline 23 & OC & 0.17 & 0.03 & 0.14 & 1.05 & 0.44 & 0.95 & 0.18 & 0.15 & 0.38 \\
\hline 24 & DME & 7.66 & 5.07 & 2.59 & 19.29 & 15.69 & 11.23 & 0.66 & 3.77 & 26.28 \\
\hline 25 & $G Y P^{-1}$ & 7.97 & 6.08 & 1.89 & 11.40 & 9.96 & 5.54 & 0.76 & 4.44 & 17.92 \\
\hline
\end{tabular}


Fig.1 Classification of genotypes on basis of height of first primary branch into 3 different categories

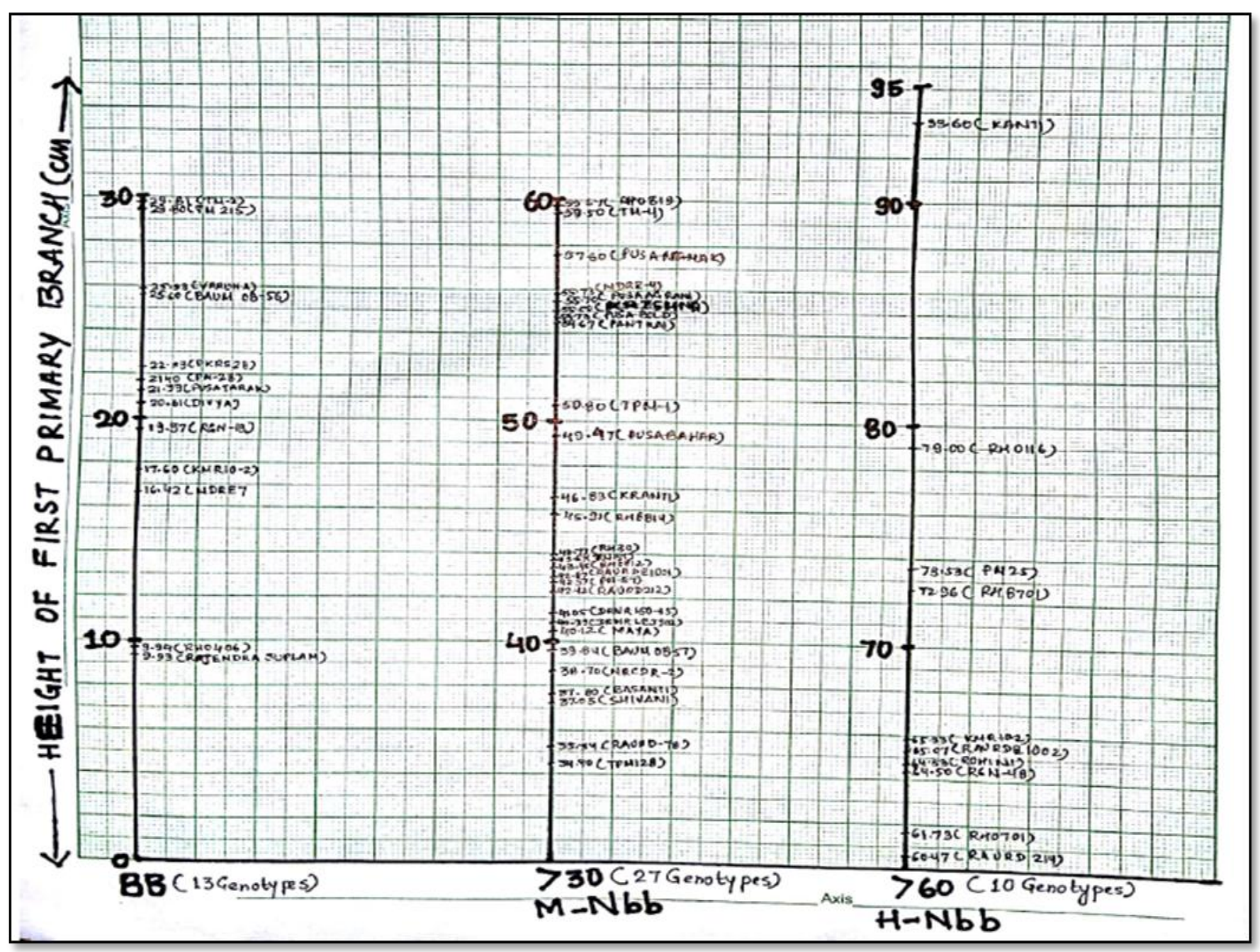

(A) Basal branching genotypes (BB) :13

(B) Medium Non-basal branching genotypes (M-Nbb) :27

(C) High Non-basal branching genotypes (H-Nbb):10 
Fig.2A Glyph -Array placement of 50 indian mustard genotypes into 9 different categories

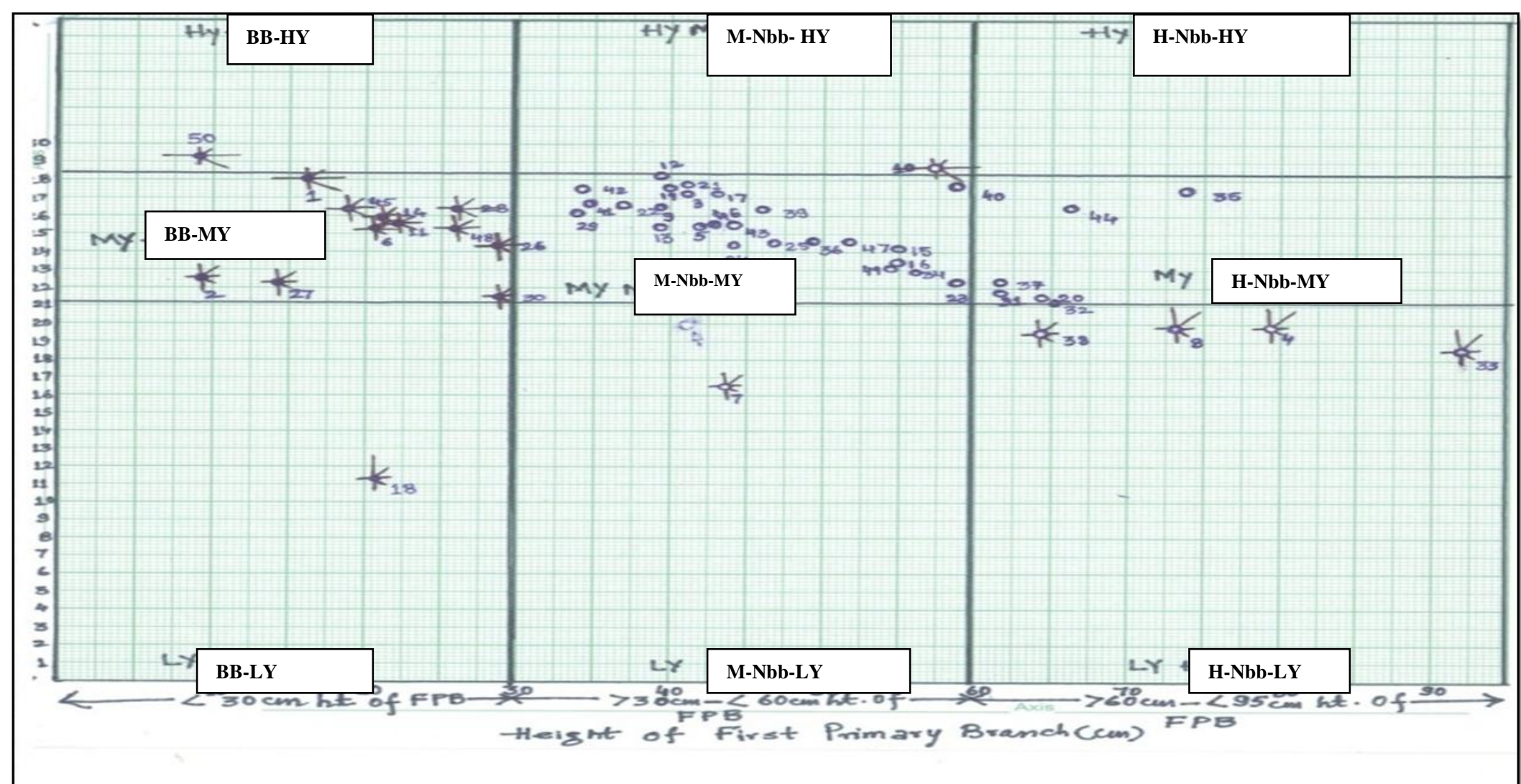

BASAL BRANCHING GENOTYPES

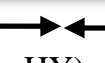

NON- BASAL BRANCHING GENOTYPES

(A) Basal Branching genotypes- High Yield - (BB- HY) :1

(B) Basal Branching genotypes- Medium Yield - (BB MY) :11

(C) Basal Branching genotypes- Low Yield (BB- LY):1

(D) Medium Non-basal branching genotypes- High Yield (M-Nbb- HY) :1

(E) Medium Non-basal branching genotypes- Medium Yield (M-Nbb- MY) :25

(F) Medium Non-basal branching genotypes- Low Yield (M-Nbb- LY) :1

(G) High Non-basal branching genotypes- High Yield (H-Nbb- HY) : -

(H) High Non-basal branching genotypes- Medium Yield (H-Nbb- MY) :6

(I) High Non-basal branching genotypes- Low Yield (H-Nbb- LY) :4 
Fig.2B Glyph - Array placement of 31 indian mustard genotypes into 2 different categories

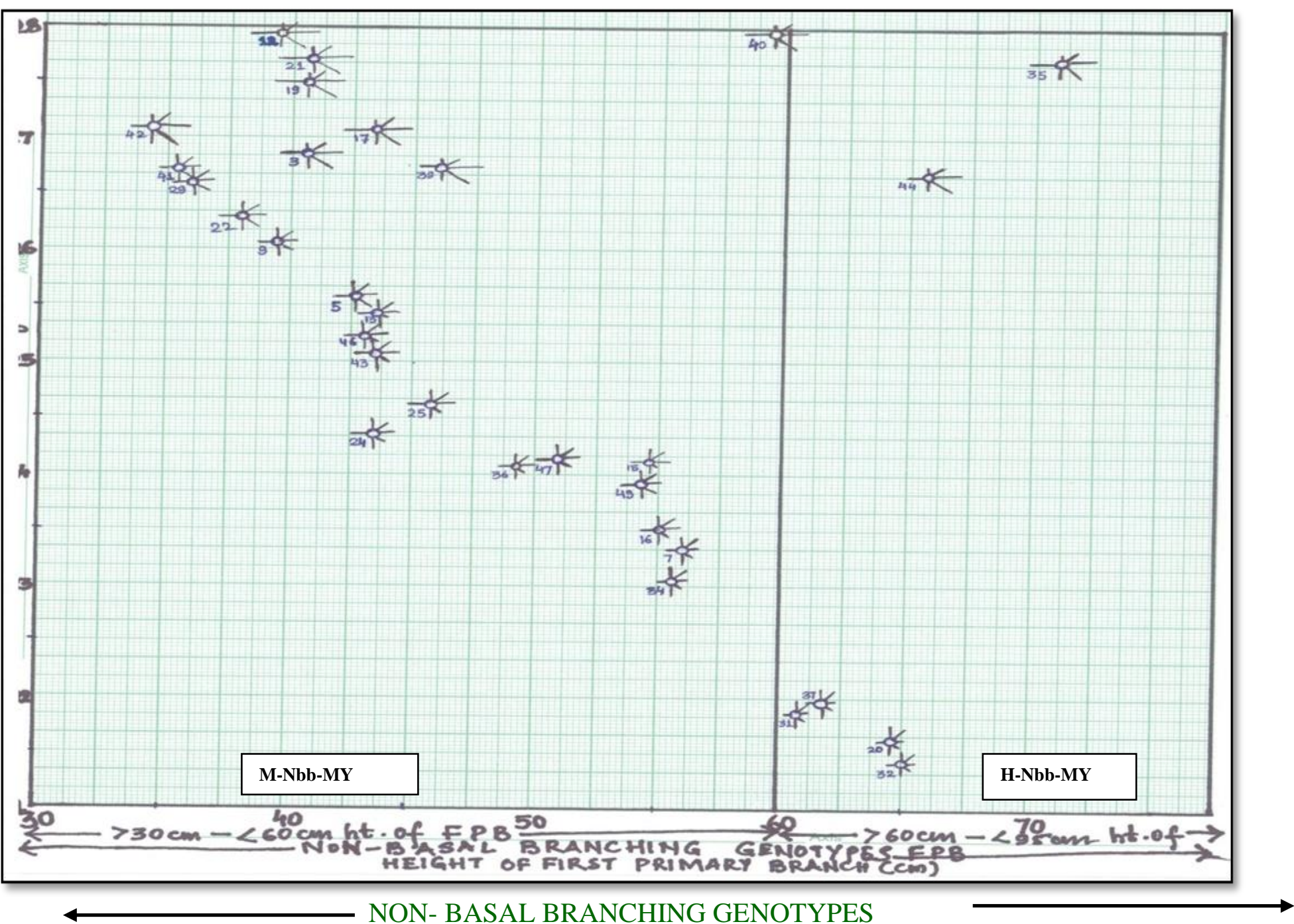

(A) Medium Non-basal branching genotypes-Medium Yield (M-Nbb-MY) :25

(B) High Non-basal branching genotypes-Medium Yield (H-Nbb -MY):6 
Moreover, the important component traits namely $\mathrm{PBP}^{-1}, \mathrm{SBP}^{-1}, \mathrm{NS}, \mathrm{SG}, \mathrm{IL}, \mathrm{HP}$, SPMA, HFPB, HFS, AB, AS, RV, RL, RG, $\mathrm{HI}$ and DME revealed high heritability coupled with high genetic advance under selection again proved the significant of unique characters like HFPB, HFS, AB, AS, RV, RL, RG and IL for developing early, dry matter efficient genotypes with high harvest index for rainfed agro ecosystem of mustard cultivation in Bihar.

Characterization of varieties and strain also provides quantum of variability for specific traits like branching behavior, root and siliqua characteristics etc. in particular given environment like rainfed condition. The genetic variability, heritability, genetic advance and expected genetic advance as a percent of mean for various traits were presented in (Table 6).

The GCV estimates had close agreement with PCV estimates for characters like DFFO, DFF, DPM, $\mathrm{PBP}^{-1}, \mathrm{SBP}^{-1}$, NS, LS, SG, IL, HP, SPMA, HFPB, HFS, AB, AS, RV, RL, $\mathrm{RG}, \mathrm{HI}, \mathrm{OC}$ and $\mathrm{GYP}^{-1}$ indicating that these characters were mostly governed by genetic factors as the role of environment deviating the expression of these traits was meager. Similar results in agreement with Misra (2012). An environmental factor strongly affects traits like $\mathrm{SS}^{-1}, \mathrm{BY}$ and $\mathrm{DME}$ as it evident from large difference between estimates of GCV and PCV. Similar results reported by Dipti et al., (2016) and Synrem et al., (2014).

High heritability for traits like DFF, $\mathrm{PBP}^{-1}$, $\mathrm{SBP}^{-1}$, NS, LS, SG, IL, HP, SPMA, HFPB, HFS, AB, AS, RV, RL, RG, HI, DME and $\mathrm{GYP}^{-1}$ implicated high magnitude of heritable portion of variation that could be exploited for selection of superior genotypes on basis of phenotypic performance. Similar results reported by Prasad et al., (2001).
High heritability coupled with high genetic advance was observed for NS, HP, HFPB, HFS and HI which were indicative of preponderance of additive gene action for expression of these traits, hence are amenable for simple selection.

Similar results were observed by Acharya and Pati (2008), Upadhayay and Kumar (2009) and Yadav et al., (2011).

High heritability with moderate genetic advance for traits likeSBP ${ }^{-1}$, SPMA, AB, AS and $\mathrm{RV}$ indicated that along with additive gene action non- additive gene action might be important for these characters. High heritability coupled with low genetic advance for characters like LS, SG, IL, RL, RG, DME and $\mathrm{GYP}^{-1}$ thus the non-additive gene action might be important for these traits.

Low heritability coupled with low genetic advance for traits $\mathrm{SS}^{-1}, \mathrm{TSW}$ and $\mathrm{OC}$ indicating that these traits were highly influenced by environment and selection on basis of these characters would be ineffective and improvement for these characters depends on breeding method which provides adequate progeny testing. Similar findings in agreement with Zebarjadi et al., (2011)

Various researchers based on plant type concept (Donald 1962 a,b) proposed Brassica ideotype (Mahrotra et al., 1986; Mendham, 1980; Daniels and Scarisbrick, 1983) in favourable timely- sown irrigated environments of Brassica napus and Brassica juncea. The present study under rainfed condition of Indian mustard studied genotypes reflected importance of basal branching behaviour over non-basal branching behaviour and therefore, two genotypes namely, Rajendra Suphlam and NDRE7 reflected highest yield and superiority in flowering, maturity, root, shoot, siliqua, grain and ultimately the yield. 
A perusal of Table 5 reflected importance of earliness for flowering and maturity deep tap root with high root, root girth, lower placement of siliqua from the base of the plant i.e., HFS, NS, SL, PH, SS ${ }^{-1}$, TSW, HI and DME. Basal branching behaviour reflected superiority of primary basal branches (more no 4-6), secondary basal branches (more number approx.10-12), less internode $(5-6 \mathrm{~cm})$ and bolder seeds with high TSW (6.5-7.5g) as compared to that to more basal branches for above traits. Siliqua characteristics under rainfed condition clearly exhibited the importance NS and $\mathrm{SS}^{-1}$ along with high seed weight. These results are in conformity to that of the findings of Mendham, 1980 (more SS ${ }^{-1}$ and high TSW); Mehrotra et al., 1976 (more pods, SS ${ }^{-1}$ and high BY). Importance of HI as observed in the present investigation was in accordance with finding of Thurling, 1992 who suggested it as best selection criteria under various environments. He also suggested careful consideration regarding BY and dry matter for development of plant type. The observations clearly indicated superiority of HI (a ratio of Economic yield and Biological yield; Donald and Hamblin, 1976) and DME (reflecting per day production as it is derived from formula HI divided by DPM) as we have noticed the importance of earliness in flowering and maturity under rain dependent Brassica management in the high yielding basal (Rajendra Suphlam and NDRE7) and non- basal branching genotypes (Pusa Mahak, NRCDR-2 and RH0819). Thurling (1947 a and b) and Gallagher et al., 1975 stated the importance of root - growth and development studies in Brasssica improvement. Noteworthy is the importance reflected by root parameters (RL, RV and RG) in rainfed Brassica genotype.

Out of these high heritability and GA under selection was reflected by NS, HP, HFPB (i.e., Basal branching type), HFS (i.e., lower siliqua placement from the base of the plant) and HI. Further, emphasized the importance of above mentioned traits. However, root volume reflected high heritability with moderate GAM. Conclusively, for rainfed situation Brassica juncea genotype with more primary basal branch (4-6) accommodating atleast two secondary basal branch per primary branches, less no. of primary and secondary $\mathrm{Nbb}$ with lower placement of first siliqua from base of plant, HP, superior in root parameters (RV), more NS and other yield attributing component traits namely $\mathrm{HI}$ and DME. Important traits likes, $\mathrm{SS}^{-1}$, TSW (low heritability and GAM) and RL, LS, RG (high heritability and low GAM) need careful consideration due to more importance of nonadditive gene action and also under the influence of critical studied environment i.e, rainfed condition.

\section{Acknowledgement}

Authors are thankful to ICAR-Directorate of Rapeseed and Mustard Research, Bharatpur, Rajasthan for providing genotypes of rapeseed and mustard.

\section{References}

Acharya, N.N. and Pati, P. 2008. Genetic variability, correlation and path analysis in Indian mustard (Brassica juncea L.). Environment and Ecology, 26 (4B): 2165-2168.

Burton, G.W. (1952). Quantitative Inheritance in Grasses. Proc. $6^{\text {th }}$ Int. Grassland Congress. 1: 227-283.

Burton, G.W. and De Vane (1953). Estimating heritability in tall Fescue from replicated clonal material. Agron. J., 45: 475-481.

Burton, W.A., Pymer, S.J., Salisbury, P.A., Kirk, J.T.O. and Oram, R.N. (1999). Performance of Australian canola quality Brassica juncea breeding lines. 
In: Wratten N, Salisbury PA (Eds), $10^{\text {th }}$ International Rapeseed Congress, pp.113-115.

Campbell, C. A., Zentner, R. P., McConkey, B. and Selles, F. 1992. Effect of nitrogen and snow management on efficiency of water use by spring wheat grown annually on zero-tillage. Can. J. Plant Sci. 72: 271-279.

Daniels. R.W. and Scarisbrick, D.H. 1983. Oilseed rape physiology in N.A.C. cereal unit, yield of oilseed rape-course papers.pp.29-46.

Dhanwani, K.R., Sarawgi, A.K., Solanki, A. and Tiwari, J.K.2013.Genetic variability analysis for various yields attributing and quality traits traits in rice (Oryza sativa L.). The Bioscan.8 (4): 14031407.

Dipti, Chawla, V., Priyanka. 2016. Correlation and path coefficient analysis for yields contributing parameters in Indian mustard [Brassica juncea (l.) Czern \& coss.]. Am. J. Pharm Tech Res. 6 (2). 655-675

Donald, C.M. 1962a. In search of yield.Journal of Australian Institute Agricultural Science.28:171-178.

Donald, C.M. 1962b. The breeding of crop ideotypes. Euphytica. 17: 385-403.

Donald, C.M. and Hamblin, J. (1976). The biological yield and harvest index of cereals as agronomic and plant breeding criteria. Adv. Agron., 28: 361-405.

Gallagher, J.N., Biscoe, P.V and Scott, R.K. 1975. Barley and its Environment V. stability of grain weight. Journal of Applied Ecology. 12: 319-324.

Ghosh, R.K. and Chatterjee, B.N. (1998). Effect of dates of sowing on oil content and fatty acid profiles of Indian mustard. Indian J. Oilseed Res., 5 (2), 144-149.

Iraddi, V.S. (2008). Response of Mustard [Brassica juncea (L.) Czernj and Cosson] varieties to date of sowing and row spacing in northern zone of Karnataka. Thesis of MSc, January 2008. College of Agriculture, University of Agricultural Sciences, Dharwad, p. 81.

Johnson, H. W., Robinnson, H. F. and Comstock, R. E. (1955). Genotypic and phenotypic correlation in soybean and their implication in selection. Agron. J., 47: 447-483.

Joshi, A. H. and Dhawan, N. L. 1966.Genetic improvement of yield with special reference to self-fertilizing crop. Indian J. Genet.Pl. Breed.26A: 101-113.

Lush, J. L. (1949).Inter-se, correlation and regression of characters. Proc. Am. Soc. Anim. Prod., 33: 293-301.

Md. Mukhlesur, R., Y. Hirata, and Fuchu-shi. (2004). Genetic diversity in Brassica species using SDS-PAGE analysis. Journal of Biological Sciences 4 (2): 234-238.

Mehrotra, O.N., Saxena, H.K. and Moosa, Mohd.1976. Physiological analysis of Varietal differences in seed yield of Indian mustard. ((Brassica juncea L. Czern \& Coss)). Indian journal of Plant Physiology. 19: 139-146.

Mendham, N.J. 1980. Crop physiology of rapeseed. Proceedings of Seminar in the E.E.C. Programme of coordination of research improvement of the production of the plant proteins. Pp. 17 (Edited by E.S. Bunting).

Misra, A.K. 2012. Genetic variability and correlation studies on germplasm of yellow sarson (B. rapa L. var. yellow sarson) for seed yield and its component traits. Cruciferae Newsletter vol. 30. Pp 46-50

Nevo, E., Golenberg, E., Beilies, A., Brown, A.H.D. and Zohary, D. 1982. Genetic diversity and Environmental associations of wild wheat in Israel. Theor.Appl.Genet. 62: 241-254. 
Prasad, L., Singh, M. and Dixit, R.K. (2001). Analysis of heritability and genetic advance in Indian mustard [Brassica juncea (L.) Czern and Coss.]. Adv. Plant Science.14 (2): 577-581.

Robertson MJ, Holland JF. 2001. Production risk of canola in the semi-arid subtropics of Australia. Australian Journal of Agricultural Research, 55: $525-538$.

Synrem G, Rangare N, Myrthong I, Bahadure D. Variability studies in Intra specific crosses of Indian mustard [Brassica juncea (L.) Czern and Coss.] genotypes. IOSRJAVS. 2014; 7 (9):29-32.

Thurling, N. 1992: Physiological constraints and their genetic manipulation. In: K.S. Labana, S.S. Banga and S.K. Banga (eds.) Breeding Oilseed Brassicas, pp. 44-46. Narosa Publishing House, New Delhi.
Upadhyay, D. K. Kumar, K. (2009) Analysis of heritability and genetic advance in relation to yield and its components in Indian mustard (Brassica juncea $\mathrm{L}$. Czern and Coss) under normal and late sown conditions. International Journal of Plant Sciences (Muzaffarnagar) 4(1): $12-14$

Yadava, D.K., Giri, S.C., Vignesh, M., Vasudev Sujata; Yadav, Kumar, Anil; Dass, B., Singh, Rajendra; Singh, Naveen; Mohapatra, T. and Prabhu, K.V. 2011. Genetic variability and trait association studies in Indian mustard (Brassica juncea). Indian Journal of Agricultural Sciences, 81 (8): 712-716.

Zebarjadi, A. Kakaei, M. and Mostafaie, A.2011.Genetic variability of some traits in Rapeseed (Brassica napus L.) under drought stress and non-stress conditions. Biharean Biologist 5 (2): pp. $127-131$.

\section{How to cite this article:}

Khushboo Chandra, Anil Pandey and Mishra, S.B. 2018. Characterization and Genetic Variability of Indian Mustard Genotypes for Branching Behavior, Yield and Its Attributes under Rainfed Condition. Int.J.Curr.Microbiol.App.Sci. 7(06): 828-846. doi: https://doi.org/10.20546/ijcmas.2018.706.098 